\title{
Processing of place information in stop consonants
}

\author{
JAMES R. SAWUSCH \\ State University of New York at Buffalo, Buffalo, New York 14226
}

\begin{abstract}
One of the basic questions that models of speech perception must answer concerns the conditions under which various cues will be extracted from a stimulus and the nature of the mechanisms which mediate this process. Two selective adaptation experiments were carried out to explore this question for the phonetic feature of place of articulation in both syllableinitial and syllable-final positions. In the first experiment, CV and VC stimuli were constructed with complete overlap in their second- and third-formant transitions. Despite this essentially complete overlap, no adaptation effects were found for a VC adaptor and a $\mathrm{CV}$ test series (or vice versa). In the second experiment, various vowel, vowel-like, and VC-like adaptors were used. The VC-like adaptors did have a significant effect on the CV category boundary, while the vowel and vowel-like stimuli did not. These results are interpreted within both one- and twolevel models of selective adaptation. These models are distinguished by whether selective adaptation is assumed to affect a single auditory level of processing or to affect both an auditory level and a later phonetic level. However, both models incorporate detectors at the auditory level which respond whenever particular formant transitions are present. These auditory detectors are not sensitive to the position of the consonant transition information within the syllable.
\end{abstract}

In the area of speech perception, many of the recently proposed models of perception have employed the concept of feature detectors. The primary empirical support for this concept has come from experiments involving selective adaptation. In the selective adaptation paradigm, repeated exposure to certain speech or speech-like sounds has been found to systematicaly alter the perception of a speech syllable series. This result, first obtained by Eimas and his coworkers for the phonetic feature of voicing (Eimas, Cooper, \& Corbit, 1973; Eimas \& Corbit, 1973) and by Cooper (1974) and Bailey (Note 1) for the place feature has led investigators to propose feature detectors that are sensitive to certain critical aspects of the speech signal. For the place feature in initial stop consonants, these aspects appear to be the second- and third-formant transitions (Bailey, 1975; Tartter \& Eimas, 1975) and the presence of an initial noise burst (Diehl, 1975; Ganong, Note 2). These are precisely the acoustic aspects of the speech signal which have previously been found to be sufficient cues for identifying the place feature in the stop consonants (Cooper, Delattre, Liberman, Borst,

The research reported here was supported by National Institute of Mental Health Grant MH-24027 and National Institutes of Health Grant NS-12179 to Indiana University. The author would like to thank Dr. Kennedth Stevens of MIT for helpful comments and discussion during the course of these experiments and Dr. David B. Pisoni for his advice and comments during all phases of this work. The research reported here was part of a doctoral dissertation submilted to Indiana University.
\& Gerstman, 1952; Hoffman, 1958; Liberman, Delattre, Gerstman, \& Cooper, 1956).

The models of the perceptual system that have been proposed to account for the selective adaptation data generally fall into two categories: one level or two levels of processing. The one-level models have sought to explain the adaptation results as originating from an auditory level of processing that is sensitive to specific characteristics of the speech signal (Ades, 1975; Bailey, 1975). Bailey (1975) has characterized this level as one corresponding to a neural spectrogram in its representation of the speech signal. Selective adaptation would systematically alter this representation, possibly causing part of it to become weaker. While the one-level models seem to be capable of explaining a large part of the current selective adaptation data, there are data which the one-level models have difficulty in dealing with (see Sawusch, 1977; Ganong, Note 2). In these experiments, adaptors were chosen so that they did not share any spectral components with the test series. However, both Sawusch (1977) and Ganong (Note 2) have found adaptation with these types of stimuli for the place of articulation dimension. These data have been interpreted as supporting the involvement of two levels of processing in the selective adaptation of speech.

A number of investigators have proposed two-level models of selective adaptation (Blumstein, Stevens, \& Nigro, 1977; Cooper, 1975; Sawusch, 1977; Tartter \& Eimas, 1975). These models have incorporated an auditory level, similar to that involved in the one- 
level models, and a second, abstract level of processing which deals with patterns of events in the speech signal (cf. Stevens, Note 3). This second level, often labeled phonetic, has also been proposed to be susceptible to selective adaptation.

One crucial question can be asked, however, regardless of which model is eventually adopted. This question concerns the range over which the adaptation phenomena will take place. This question has been addressed in a number of ways. For the place feature, experimenters have manipulated the spectral overlap between adaptor and test series (Bailey, 1975; Sawusch, 1977); they have manipulated the burst and transition cues separately (Blumstein, Stevens, \& Nigro, 1977; Diehl, 1975; Ganong, Note 2); they have used various nonspeech adaptors (Pisoni \& Tash, 1975; Tartter \& Eimas, 1975) and speech adaptors with different phonetic composition (Cooper, 1974; Cooper \& Blumstein, 1974); and they have manipulated the position of the place information within the syllable (Ades, 1974; Pisoni \& Tash, 1975; Pisoni, Note 4). This last type of manipulation, involving the transfer of adaptation from a CV to a VC context (and vice versa) has led to divergent claims about the range over which selective adaptation to place will occur.

Ades (1974) attempted to selectively adapt a CV series ([bae]-[dae]) with mirror-image VC syllables ([aeb] and [aed]), and vice versa. The results of this experiment showed no cross-series adaptation. The VC stimuli exhibited no adapting effect on the CV test series, and, conversely, the CV adaptors had no effect on the VC test series. Ades $(1974,1976)$ has interpreted these results as demonstrating that adaptation was not occurring at a level where the phonetic feature of place is represented independently of syllable position. Further, within the context of a one-level model of adaptation, Ades (1976) has proposed that different mechanisms mediate consonant place perception for the initial vs. final stops, at least at the particular level affected by selective adaptation. On theoretical grounds, this interpretation is somewhat discrepant with an auditory feature detector interpretation of selective adaptation. If Ades' (1976) interpretation is correct, then feature detector models will have to add separate sets of detectors that respond to much the same information, but in different syllable positions. Potentially, this could lead to an almost infinite number of feature detectors and render this whole concept useless (see Weisstein, 1973). However, as noted by Pisoni and Tash (1975), some care must be exercised in interpreting the results of Ades' (1974) CV-VC transfer experiment.

Pisoni and Tash (1975) of fered two alternative explanations for the failure of a VC syllable to adapt a CV series (and vice versa). One possibility was that an insufficient spectral match existed between the consonant portions of the $\mathrm{CV}$ and $\mathrm{VC}$ syllables. The formant transitions for the consonantal portions of these syllables in Ades' (1974) experiment had curvilinear trajectories, as shown in Figure 1. Consequently, while the second- and third-formant trajectories for the [bae] end of the CV series exhibit a negative acceleration over time, the corresponding formants in the [aeb] syllable exhibit a positive acceleration of frequency change over time. In an attempt to correct for this, Pisoni (Note 4) employed $\mathrm{CV}$ and VC stimuli with linear formant transitions. A small, but consistent, adapting effect was found for the VC adaptors on the CV test series, although the direction was opposite that which would be expected on a phonetic basis. This question of direction will be returned to later. It should be noted that even if the formant transitions in Ades' (1974) stimuli had been linear, the spectral overlap between VC adaptor and CV test would still not have been extensive. Since previous experiments have shown that spectral overlap is a primary factor in adaptation (see Ades, 1976; Bailey, 1975), the negative results of Ades' (1974) CV-VC experimeni could be due to this lack of spectral overlap.

The second alternative explanation offered by Pisoni and Tash (1975) for the negative findings of Ades (1974) was that two components were involved in adapting a CV series with a VC syllable. Furthermore, these two components exhibited adaptation in opposite directions and cancelled each other out. In one of these components, termed the auditory level, adaptation is governed by the spectral overlap between the adaptor and the test series. Thus, for a [bae]-[dae] CV series, rising formants would cause [b] adaptation and falling formants would cause [d] adaptation (i.e., adaptation as if by a [d] phoneme). From an inspection of Figure 1, it can be seen that

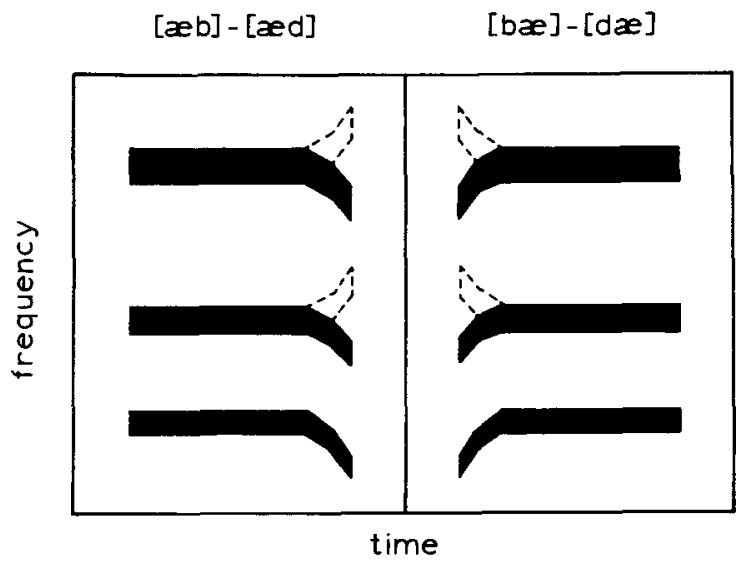

Figure 1. Schematic spectrograms of the [aeb] (solid formants) and [ae] (dashed formants) end-points and the [bae] (solid formants) and [dae] (dashed formants) end-points from these two sets of syllables. (After Ades, 1974.) 
the [aed] syllable has rising formant transitions and the [aeb] syllable has falling formant transitions. Thus, the [aed] syllable should have a [b]-adapting effect on the [bae]-[dae] test series at the auditory level. Similarly, the [aeb] adaptor should have a [d]-adapting effect on the [bae]-[dae] series at the auditory level. This is precisely the kind of result that was reported by Pisoni (Note 4), as mentioned above. In contrast, at a second, phonetic level, the adaptation effects might be expected to follow the phonetic identity of the adaptor and test series. At this level, [aeb] would [b]-adapt the [bae]-[dae] test series and [aed] would [d]-adapt this series. Consequently, in the experiment of Ades (1974), these two effects might have cancelled each other out, yielding no net adaptation effect, or left a small residual result due to one level exhibiting a larger effect than the other (as in the data of Pisoni, Note 4, with a small net auditory effect). In an experiment intended to test the auditory half of this two-level account, Pisoni and Tash (1975) employed as adaptors two speech-like stimuli termed speechembedded chirps (SEChirp). These stimuli were constructed from the end-points of a [ba]-[da] CV test series. For the " $b$ "'-SEChirp, steady-state formants were added to the beginning of the [ba] syllable with center frequencies that corresponded to the starting frequencies of the first three formant transitions. The steady-state formants following the transitions were then eliminated. The resultant " $b$ "'-SEChirp resembled a VC syllable in that the formant transitions were in final position (after the steady state). However, such a syllable would be impossible for a human to produce because of the rising final firstformant transition. (For a VC syllable, F1 falls in final position, as shown in Figure 1). A "d"'-SEChirp was constructed in a similar manner from the [da] end-point syllable. When used as adaptors, the " $b$ "SEChirp caused a shift in the [ba]-[da] category boundary toward [ba] (a [b]-adapting effect) and the " $d$ "'SEChirp caused a shift toward [da]. These are precisely the directions that would be expected on the basis of the spectral match between the formant transitions of the adapting and test stimuli. However, in terms of a phonetic level, these results are opposite what would be expected, since " $b$ "'-SEChirp more closely resembles a [d] in final position than a [b].

The failure of Ades (1974) to find adaptation of a VC stimulus on a CV series could have been due to any of the three possibilities outlined above: (1) Adaptation does not transfer from syllable initial to syllable final position (and vice versa), as suggested by Ades $(1974,1976)$; (2) there was insufficient spectral overlap between the $\mathrm{CV}$ and VC syllables; or (3) there was a cancellation between auditory and phonetic components of adaptation that went in opposite directions (Pisoni \& Tash,
1975). The results of Pisoni and Tash, using SEChirps as adaptors, would seem to rule out Explanation 1 above, since the transition information in final position (SEChirps) did cause adaptation of similar information in initial position ([ba]-[da] CV syllables). However, either or both of Explanations 2 and 3 could be correct. Experiment 1 was conducted to compare the original explanation of Ades (1974) and the cancellation explanation of Pisoni and Tash (1975) using CV and VC stimuli with maximal spectral overlap, similar to the SEChirps.

\section{EXPERIMENT 1}

The first experiment was designed to partially resolve the conflicting interpretations of the experiments of Ades (1974) and Pisoni and Tash (1975). In order to do so, separate sets of CV and VC test syllables were constructed so as to maximize the spectral overlap in formant transitions between one end of the CV series and one end of the VC series. The end-points of the two sets are shown as schematized spectrograms in Figure 2. The secondand third-formant transitions of the [bae] endpoint from the [bae]-[dae] series are identical with those of the $[\Lambda d]$ endpoint from the $[\wedge b]-[\Lambda d]$ series. Thus, these stimuli include the spectral overlap of the SEChirps used by Pisoni and Tash (1975) in a full VC context similar to that used by Ades (1974) and Pisoni (Note 4). Using these stimuli, it was hoped that further light could be shed on the questions of positional dependence of selective adaptation and cancellation of effects at multiple levels of processing.

\section{Method}

Subjects. The subjects for this experiment were 10 undergraduates at Indiana University who responded to an advertisement in the student newspaper. They were all right-handed native speakers of English with no known history of any speech or hearing disorder and were paid for their participation at the rate of $\$ 2 / \mathrm{h}$.

Stimuli. Two sets of synthetic five-formant syllables were constructed on the OVE IIId speech synthesizer at Indiana University. One series consisted of seven $C V$ syllables that ranged perceptually from [bae] to [dae]. These stimuli varied in the starting frequencies for the second- and third-formant transitions from $1,198 \mathrm{~Hz}(\mathrm{~F} 2)$ and $2,262 \mathrm{~Hz}(\mathrm{~F} 3)$ for the [bae] end of the series to $2,135 \mathrm{~Hz}(\mathrm{~F} 2)$ and $3,489 \mathrm{~Hz}(\mathrm{~F} 3)$ at the [dae] end in six approximately equal steps. The first-formant transition had a starting frequency of $251 \mathrm{~Hz}$ for all seven stimuli. The transitions were followed by a steady-state vowel [ae] that had formant center frequencies of $654 \mathrm{~Hz}$ (F1), 1,647 Hz (F2), and 2,850 Hz (F3).

The second series contained seven VC syllables that ranged perceptually from $[\Lambda b]$ to $[\Lambda d]$. These stimuli varied in their terminal frequencies for their second- and third-formant transitions. The $[\wedge b]$ end of the series had terminal frequencies of $755 \mathrm{~Hz}$ (F2) and 1,695 $\mathrm{Hz}$ (F3), while the [ $\Lambda \mathrm{d}]$ end of the series had terminal frequencies of $1,647 \mathrm{~Hz}(F 2)$ and $2,850 \mathrm{~Hz}(\mathrm{~F} 3)$. The five stimuli between these two onds had terminal frequencies that were approximately equally spaced. The first-formant transi- 
tion fell to $251 \mathrm{~Hz}$ for all seven stimuli. The formant transitions for all seven stimuli were preceded by a steady-state vowel resembling $[\Lambda]$ with formant center frequencies of $645,1,198$, and $2,265 \mathrm{~Hz}$. Stylized sound spectrograms of the end-points of these two series are shown in Figure 2. All formant transitions for both series had a duration of $45 \mathrm{msec}$ and the vowel duration of each syllable was $205 \mathrm{msec}$. The fundamental frequency for all 14 syllables was held constant at $118 \mathrm{~Hz}$. Finally, to minimize certain onset and offset transients, the envelope amplitude of the seven $\mathrm{CV}$ syllables was linearly ramped of over a period of $50 \mathrm{msec}$. Similarly, the envelope amplitude of the seven VC syllables was linearly ramped on over a 50 -msec period.

One final stimulus, to be used as an adaptor, was also constructed. An F2F3-chirp was produced on a parallel resonance synthesizer at MIT.' This two-formant chirp had a duration of $45 \mathrm{msec}$ and was constructed to match the second- and thirdformant transitions of the [bae] end-point of the [bae]-[dae] series and the $[\Lambda \mathrm{d}]$ end-point of the $[\Lambda \mathrm{b}]-[\Lambda \mathrm{d}]$ series. This chirp had initial frequencies of 1,198 and $2,262 \mathrm{~Hz}$ and final frequencies of 1,646 and $2,850 \mathrm{~Hz}$ for its two formants. As in the CV and VC series, the fundamental frequency was constant at $118 \mathrm{~Hz}$. This chirp was recorded on magnetic tape and later digitized and stored on the disk memory of a PDP -11 computer.

Procedure. All experimental events were controlled by a PDP-11 computer. The stored parameter codes for the OVE were synthesized in real time and presented binaurally to subjects through Telephonics (TDH-39) matched and calibrated headphones. The digitized F2F3-chirp was reconverted to analog form in real time (via 12-bit D/A converter) and also presented binaurally to subjects. The stimuli were presented at a level of $80 \mathrm{~dB}$ SPL for a steady-state calibration vowel [ae].

The experiment consisted of three $1-h$ sessions that were run on consecutive days. The subjects were run in two groups of five subjects each. One of these groups was always tested on the [bae]-[dae] series and the other was always tested on the $[\wedge b]-[\wedge d]$ series. On the first day only, the subjects were given a 35-trial practice sequence in which each of the seven syllables in their test series was presented five times in random order. Next, the subjects listened to a 70 -item identification test, with each syllable occurring 10 times in random order. The subjects were instructed that they would be hearing the syllables [bae] and [dae] $([\wedge \mathrm{b}]$ and $[\wedge d])$ and were to identify each syllable as either $[$ bae] or [dae] or $([\Lambda b]$ or $[\Lambda d])$. Following their identification response, the subjects were instructed to enter a rating response, indicating how certain they were that they had identified the syllable correctly. A 4-point scale was used in which a 1 denoted that the subject was positive the response was correct, a 2 denoted the subject throught that it was probable that the response was correct, a 3 denoted that it was possible the response was correct, and a 4 denoted that the subject was guessing. A copy of the rating scale was present in front of the subjects at all times. The subjects entered both identification and rating responses by pushing the appropriate buttons on a response box in front of them.

Following the identification sequence, the subjects listened to two adaptation sequences on each day. Three adapting stimuli were used: the [bae] end-point, the [ $\wedge \mathrm{d}]$ ] end-point, and the F2F3chirp. A different adaptor was used each day, and their order was the same for both groups: [bae] the first day, $[\Lambda \mathrm{d}]$ the second day, and the F2F3-chirp on the third day. In all cases, the subjects were informed as to the exact nature of the repeated sound that they would be listening to.

In a block of adaptation and testing, the adaptor was presented 75 times with $300 \mathrm{msec}$ between presentations. Following this period of adaptation, the seven test stimuli were presented in random order for identification and rating by subjects. Twenty of these adaptation sequences were run on each day. Thus, by the end of the experiment, each subject had provided 30 unadapted responses and 20 adapted responses (for each adaptor) to each of the seven test syllables in their series.

\section{Results and Discussion}

Rating functions for each subject were obtained by expanding the two separate category responses with their associated 4-point rating responses into an 8 -point rating scale. On this scale, a 1 denotes a positive rating for a " $b$ " response. At the other end, an 8 denotes a positive rating for a " $d$ " response. The phonetic boundary was determined by a computer program that located the point along the stimulus continuum corresponding to a rating of 4.5 by linear interpolation. For both the [bae]-[dae] and the $[\wedge b]-[\wedge d]$ series, the end-point adaptor from the test series had a significant adapting effect. The [bae] adaptor moved the [bae]-[dae] category boundary toward [bae] $[t(4)=3.95, p<.01]$ and the $[\Lambda d]$ adaptor shifted the $[\Lambda \mathrm{b}]-[\Lambda \mathrm{d}]$ category boundary toward $[\Lambda \mathrm{d}][\mathrm{t}(4)=6.70, \mathrm{p}<.005] .{ }^{2}$ The F2F3chirp also had a significant effect on both series, moving the [bae]-[dae] boundary toward [bae] [t(4) $=2.79, \mathrm{p}<.05]$ and the $[\wedge \mathrm{b}]-[\wedge \mathrm{d}]$ boundary toward [^d] $[t(4)=2.62, p<.05]$. The average unadapted rating function for the [bae]-[dae] test series group is shown in the left panel of Figure 3 along with the [bae] and the F2F3-chirp adapted functions. The left panel of Figure 4 shows the baseline, [ $\wedge d$ ] adapted, and F2F3-chirp adapted rating functions for the $[\Lambda \mathrm{b}]-[\Lambda \mathrm{d}]$ test series group.

In contrast to these within-series adaptation conditions, no category boundary shifts were found in the cross-series conditions. The right panel of Figure 3 shows the [bae]-[dae] rating function before and after adaptation with $[\wedge d]$. The curves are virtually identical, and no significant boundary shift was found. The same pattern of results can be seen in the right panel of Figure 4 for [bae] adaptation and testing on the $[\wedge b]-[\Lambda d]$ series. No significant category shift was found. ${ }^{3}$

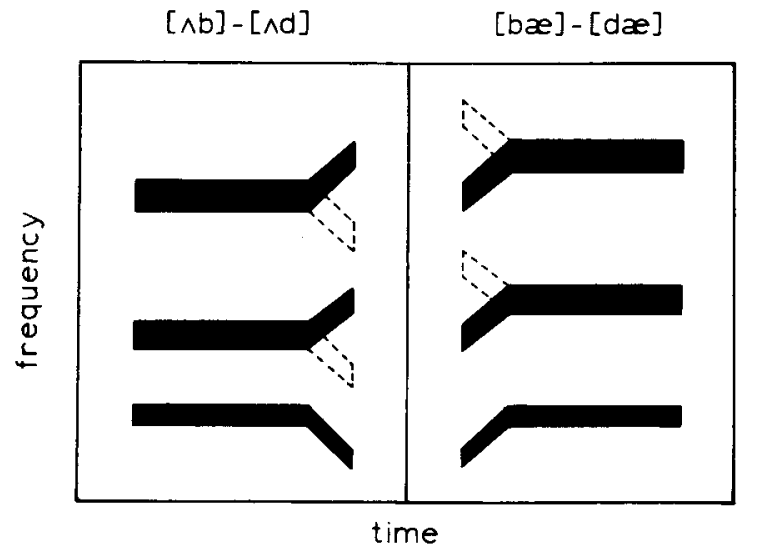

Figure 2. Schematic spectrograms of the $[\wedge b]$ (dashed formants) and $[\Lambda \mathrm{d}]$ (solid formants) end-points and the [bae] (solid formants) and [dae] (dashed formants) end-points from the two syllable sets in Experiment 1. 
[bæe] and F2F3 Adaptors

[^d] Adaptors
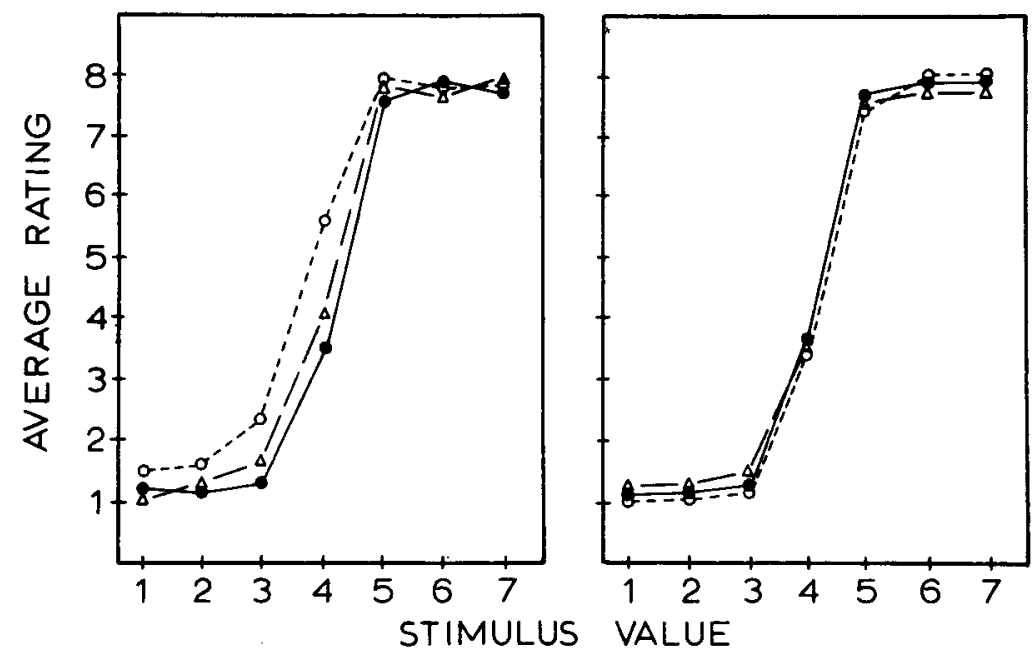

Figure 3. Unadapted (solid circles) and adapted rating functions for the [bae][dae] group. On the left are [bae] (open circles) and F2F3-chirp (open triangles) adapted functions. On the right are [ $\wedge \mathrm{d}]$-adapted functions for 75 (open circles) and 100 (open triangles) repetitions of $[\Lambda d]$.

These results agree very well with those of Ades (1974) in that no effect of a VC on a CV series (or vice versa) was found. This result was found by Ades (1974) when the VC and CV syllables were mirror images and in the present study when the spectral overlap between the transitions for VC and $\mathrm{CV}$ syllables was maximized. Consequently, the spectral overlap explanation offered by Pisoni and Tàsh (1975) for the negative results of Ades (1974) can be rejected. It would seem that the only alternative left is the cancellation explanation offered by Pisoni and Tash (1975). However, Ades (1976) has offered an alternative explanation for the Pisoni and Tash (1975) SEChirp adaptation results. Ades (1976) has pointed out that the onset frequencies for the steadystate portions of the SEChirps are identical to the onset frequencies for the initial transitions of the test series used by Pisoni and Tash. Thus, Ades

[Ad] and F2F3 Adaptors

[bæ] Adaptors
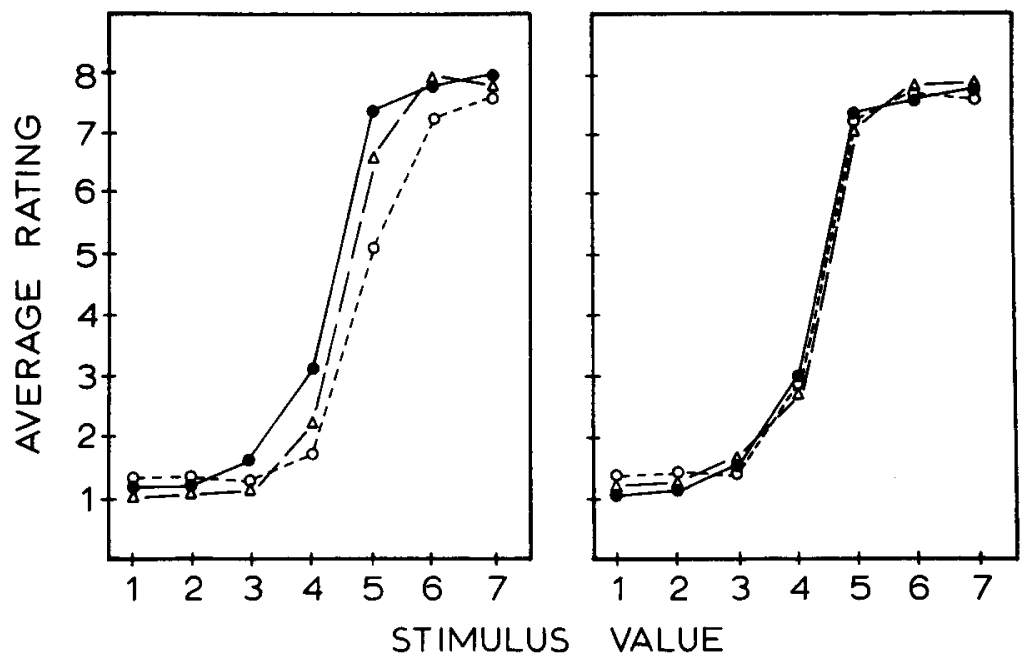

Figure 4. Unadapted (solid circles) and adapted rating functions for the $[\Lambda \mathrm{b}]-$ [ $\wedge$ d] group. On the left are [ $\Lambda$ d] (open circles) and F2F3-chirp (open triangles) adapted functions. On the right are [bae]-adapted functions for 75 (open circles) and 100 (open triangles) repetitions of [bae]. 
suggested that it was this pattern of onset frequencies of the SEChirps that was causing adaptation and not the transitions in final position. Experiment 2 was conducted as a direct test of this alternative explanation.

\section{EXPERIMENT 2}

If the initial steady-state onset of the SEChirps was causing the adaptation effect observed by Pisoni and Tash (1975), then there are still two possible explanations for the failure of a VC to adapt a CV test series (Ades, 1974; Experiment 1). Briefly, they involve either a dependence of adaptation on the position of the critical information within the syllable (Ades, 1974, 1976) or on the cancellation of two effects in opposite directions (Pisoni \& Tash, 1975). In order to explore these two explanations and the alternative offered by Ades (1976) to explain the SEChirp adaptation results, five new adapting stimuli were constructed. Four of them are shown as schematized spectrograms in Figure 5. A SEChirp similar to that used by Pisoni and Tash, was constructed from the [bae] end of the [bae]-[dae] series used in Experiment 1. A second SEChirp, labeled F2F3-SEChirp, was constructed with a constant Fl and no final rising transition, to explore the role of the rising F1 in the " $b$ "-SEChirp adaptor. To explore the explanation of Ades (1976) that the steady-

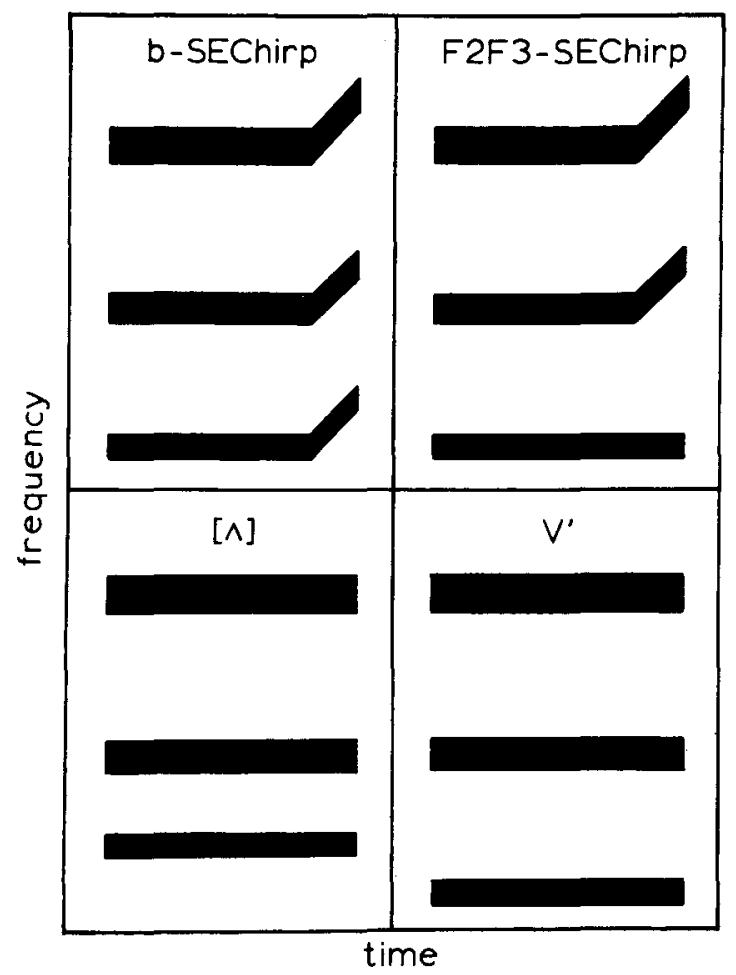

Figure 5. Schematic spectrograms of four of the adapling stimuli used in Experiment 2. state onsets were causing the SEChirp adaptation effect, two further vowel-like stimuli were constructed. Both were three-formant steady-state patterns, the only difference being the center frequency of the first formant. Both of these stimuli had an abrupt onset in envelope amplitude similar to the onset of the [bae]-[dae] test series. A fifth adaptor, consisting of wide-band noise with a $10-\mathrm{kHz}$ upper frequency limit was also used to check for any overall change in the rating responses of subjects due to repeated presentation of any acoustic stimulus. These various adaptors therefore permit a strong test of the role of onset amplitude in determining selective adaptation effects and whether adaptation is sensitive to the syllable position of the critical information.

\section{Method}

Subjects. The subjects were 30 new paid volunteers. Some of the subjects had limited previous experience with synthetic speech. All subjects met the usual requirements and were paid at the rate of $\$ 2 / \mathrm{h}$.

Stimuli. The seven step [bae]-[dae] series from Experiment 1 was also used in this study. Four additional adapting stimuli were also constructed on the OVE synthesizer. A "b"-SEChirp was constructed from the [bae] end of the test series by placing 205msec-duration steady-state formants in front of the formant transitions. These three formants had center frequencies of $251,1,198$, and $2,262 \mathrm{~Hz}$, respectively. The steady-state formants following the transitions were then deleted leaving a $250-\mathrm{mse}$ stimulus. A second SEChirp, labeled the F2F3-SEChirp, was constructed from the "b"'SEChirp. The final formant transition for the first formant was removed and replaced by a steady state formant at $251 \mathrm{~Hz}$. Schematic spectrograms of the two SEChirps appear in the top half of Figure 5. The overall envelope amplitude for both of the SEChirps was gradually ramped on over the first $50 \mathrm{msec}$ of the initial steady state.

Two vowel-like stimuli were also constructed from the [bae] end of the [bae]-[dae] series. One of these, labeled " $V$ " in Figure 5, had steady state values of $251,1,198$, and $2,262 \mathrm{~Hz}$ for the first three formants. These values are identical to the starting frequencies of the three formant transitions for the [bae] end of the [bae]-[dae] series. When synthesized, this stimulus had a distinct tonal quality and sounded something like the vowel [u]. A second vowel, labeled $[\Lambda]$ in Figure 5, had formant center frequencies of $653,1,198$, and $2,262 \mathrm{~Hz}$. These values matched the first-formant steady-state of the $[\Lambda]$ vowel to the first-formant steady state of the [ae] portion of the [bae]-[dae] test stimuli. The second- and third-formant frequencies of the $[\Lambda]$ vowel, like the stimulus $V^{\prime}$, were identical to the onset frequencies of the second-and third-formant transitions at the [bael end of the [bae]-[dae] test series. Both vowels had a duration of $250 \mathrm{msec}$ and an amplitude onset similar to that of the [bae]-[dae] test series. All four of these adaptors had a constant fundamental frequency of $118 \mathrm{~Hz}$.

The noise adaptor was construcled by the repeated sampling of 250-msec segments from a noise generator (Grason-Stadler, Model 1724) with the upper frequency cutoff set at $10 \mathrm{kHz}$. Thus, the exact composition of the noise adaptor was random from presentation to presentation.

Procedure. All experimental events were controlled by a PDP-11 computer. Subjects were run in six groups of five each for one 1-h session. Each group received a different adaptor (except that two groups received the noise adaptor). The identification and test sequences were essentially identical to those of Experiment 1 except that only the [bae]-[dae] series was used for testing. Since 
subjects were run on 1 day only, the identification sequence was presented iwice betore the adaptation trials were begun. Thus. each subject provided 20 unadapted and 20 adapted respunses to caich of the seven test syllables.

\section{Results and Discussion}

The results of using the " $b$ "-SEChirp as an adaptor are shown in the left panel of Figure 6. The " $b$ "-SEChirp had a significant effect in moving the [bae]-[dae] boundary toward [bae] $[\mathrm{t}(4)=4.23$, $\mathrm{p}<.01]$. This result replicates the findings of Pisoni and Tash (1975), with one important difference. Since the steady-state portion of this adaptor was gradually ramped on over a 50 -msec period, it is unlikely that the adaptation effect was caused by the steady-state onset of the vowel. Rather, the adaptation effect seems to be due to the final transitions.

The F2F3-SEChirp had a marginally smaller adapting effect than the " $b$ "-SEChirp. The rating data for this group appear in the right panel of Figure 6. The F2F3-SEChirp also moved the [bae]-[dae] category boundary toward [bae] $[\mathrm{t}(4)=3.18$, $p<.025]$. Further, the F2F3-SEChirp and the " $b$ "SEChirp did not differ significantly in the amount of shift they induced in the category boundary. ${ }^{4}$ This indicates that the upturned first formant was not a primary contributor to the adaptation effect found by Pisoni and Tash (1975) or in the present experiment with the "b"-SEChirp. Instead, it seems to be the lack of a "normal" or falling first formant that determines whether adaptation effects take place. This point about the importance of the first formant will be considered again below.

The results of adaptation with the vowel $[\Lambda]$ and the vowel-like stimulus $\mathrm{V}^{\prime}$ appear in Figure 7. Neither adaptor had any effect on the average rating responses of subjects to the [bae]-[dae] test series. This direct test permits a rejection of Ades' (1976) claim that the vowel onset of the SEChirps could have caused the adaptation effects observed by

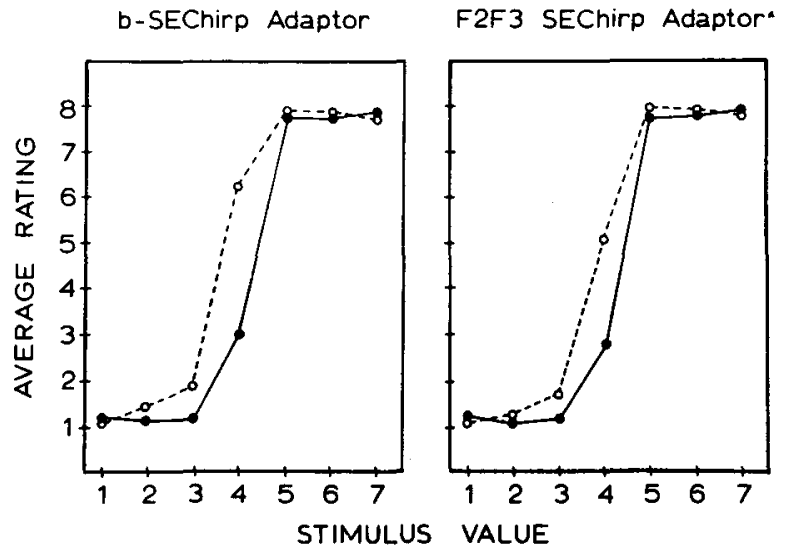

Figure 6. Unadapted (solid circles) and SEChirp-adapted (open (ircles) rating functions for the two SEChirp-adapted groups frum Experiment 2.

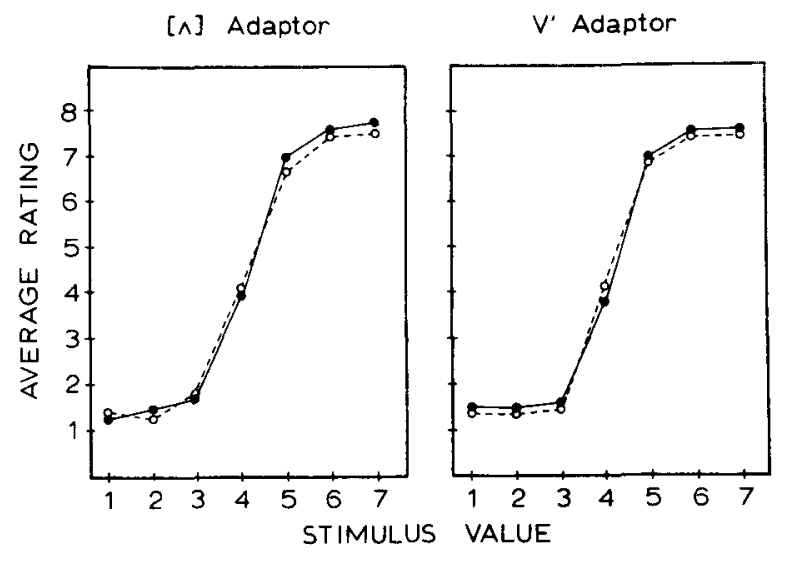

Figure 7. Unadapted (solid circles) and vowel-adapted (open (ircles) rating functions for the [bae]-[dae] series from Experiment 2.

Pisoni and Tash (1975). The formant transitions in final positions are indeed the cause of the adaptation effects observed with the SEChirps.

The lack of any adaptation effect with the vowellike stimuli also has an interesting implication in connection with a recent account of place of articulation proposed by Stevens and Blumstein (Note 5). According to these authors, the critical cue to place perception in stop consonants is the spectrum at onset (or offset). The bilabial stops $[b, p]$ are characterized by a concentration of energy at relatively low frequencies. The alveolars $[\mathrm{d}, \mathrm{t}]$ and the velars $[\mathrm{g}, \mathrm{k}]$ are characterized by concentrations at high- and middle-frequency regions, respectively. Detectors sensitive to onset (offset) would respond differentially to these relative energy concentrations. Thus, these detectors offer a potential resolution of the invariance issue for the stop-consonants (see Liberman, Cooper, Shankweiler, \& StuddertKennedy, 1967, for a review of the invariance issue). The $\mathrm{V}^{\prime}$ adaptation condition in Experiment 2 offers a direct test of this proposed detector system. The model of Stevens and Blumstein (Note 5) would characterize the $V^{\prime}$ adaptor from the present experiment as being roughly equivalent to the [bae] endpoint syllable at onset. Both of these stimuli have the same formant frequencies at onset and both have an abrupt envelope amplitude onset. Thus, one particular onset-offset detector should respond to both $V^{\prime}$ and [bae]. However, the $V^{\prime}$ adaptor had no effect on the [bae]-[dae] series, indicating that the $\mathrm{V}^{\prime}$ stimulus and the [bae]-[dae] test stimuli apparently are processed by distinct and separate mechanisms at the level(s) of processing affected by selective adaptation. Consequently, the approach taken by Stevens and Blumstein does not seem to be an adequate description of the processing at these level(s). Their account could, however, be applicable to a still later stage of analysis which is unaffected by selective adaptation. 
The final adaptation condition, where a wide-band white noise burst was used as the adaptor, also showed no adaptation. The unadapted and noiseadapted rating functions for this group are shown in Figure 8. The noise bursts had no effect on the [bae][dae] rating responses either within categories or at the category boundary. If adaptation with the noise bursts had been observed, one could argue that transition detectors were simply summing energy over time within very broad frequency regions. The absence of any effect with the noise adaptor indicates that the detectors probably do not simply sum energy. Instead, they seem to respond to particular patterns of frequencies over time. The lack of an adaptation result with wide-band noise suggests that these detectors may exhibit the inhibitory surround characteristics found for visual detectors (Hubel \& Wiesel, 1965). The inhibitory surround would mean that only a particular pattern of frequencies over time would elicit a response from such a detector. Without the inhibitory surround, a band of noise would be registered by these detectors since it would contain the appropriately spaced frequency components (as well as many adjacent, irrelevant components).

\section{GENERAL DISCUSSION: EXPERIMENTS 1 AND 2}

The overall pattern of results found in Experiments 1 and 2 seems to be quite consistent with a feature detector interpretation of selective adaptation to speech. From the SEChirp results of Experiment 2 and those of Pisoni and Tash (1975), it appears that the transition detector mechanisms at the auditory level are on "free-run." That is, they will respond whenever an appropriate frequency transition occurs, regardless of whether it is preceded by a steady state (SEChirp results of Experiment 2), followed by a steady state (from Experiment 1), or without a steady state (F2F3 from Experiment 1). Further evidence that the adaptation with the SEChirps and the F2F3-chirp takes place at an auditory level comes from the direction of the category boundary shifts. The F2F3-chirp shifted the [bae]-[dae] boundary toward [bae] but the $[\Lambda \mathrm{b}]-[\Lambda \mathrm{d}]$ boundary moved toward $[\wedge d]$. These are precisely the directions expected at a transition-specific auditory level of processing due to the spectral overlap between adaptor and test series. The shifts were in opposite phonetic directions in the two series, indicating that the locus of the effect is definitely prior to phonetic categorization. A similar line of reasoning leads to the same conclusion regarding the effect of the SEChirps (see Pisoni \& Tash, 1975). Thus, it appears that separate mechanisms for transition information in syllable-initial position vs. syllable-final

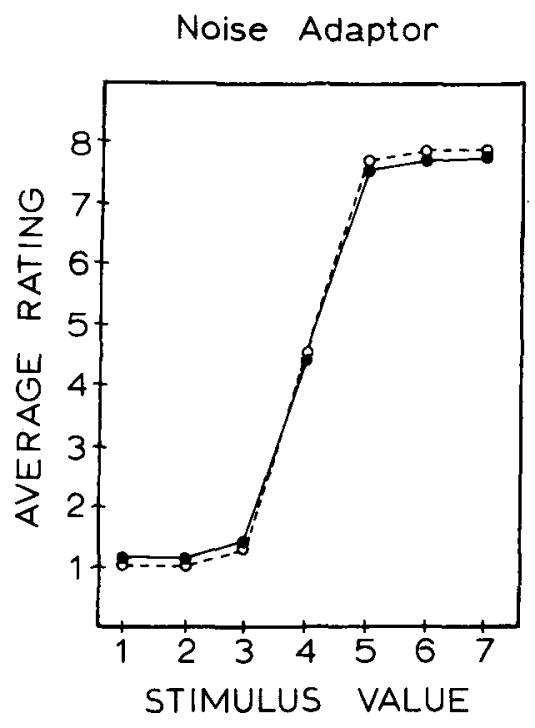

Figure 8. Unadapted (solid circles) and 250-msec wide-band noise burst adapted (open circles) rating functions for the [bael[dae] series.

position are unnecessary and a model employing feature detectors that respond whenever they detect the presence of frequency transitions is feasible.

Two explanations of the failure of a VC syllable to adapt a CV series can be ruled out on the basis of these results. The explanation offered by Ades (1974, 1976), that the mechanisms responsible for adaptation at the auditory level are sensitive to syllable position (initial vs. final), can be eliminated on the basis of the results from Experiment 2 . The alternative offered by Pisoni and Tash (1975) that the failure of a VC to adapt a CV series was due to a lack of spectral overlap can also be rejected. Experiment 1 attempted adapting a $\mathrm{CV}$ series with a VC syllable with completed spectral overlap of the important consonantal transitions. Since no adaptation effect was found, the lack of spectral overlap explanation does not seem to be supported.

At this point, the most reasonable explanation seems to be that offered by Pisoni and Tash (1975) within a two-level model of selective adaptation to place. As noted earlier, the two-level class of models has recently received additional experimental support (Sawusch, 1977). Pisoni and Tash suggested that adaptation takes place at both and auditory and phonetic levels, but that these two effects are in opposite directions and cancel. The SEChirps did not produce cancellation because they were not processed at the second (phonetic) level due to their nonspeech nature (see Pisoni \& Tash, 1975). This two-level cancellation explanation seems to fit all of the available data. However, before accepting this model fully, a demonstration of the transfer 
of adaptation from $\mathrm{CV}$ to $\mathrm{VC}$ at the phonetic level is necessary.

It also seems to be possible to account for the CV-VC results within a one-level model of selective adaptation or a two-level model in which adaptation is position-specific at the second level. In this type of model, adaptation with a VC syllable would produce an effect at the auditory level. The key question is why this effect does not show up in the responses of the subject. One possible answer lies in the differential use of the formant transitions as clues to the place feature in $\mathrm{CV}$ and VC syllables. If the secondand third-formant transitions are differentially weighted as cues in syllable-initial vs. syllable-final position, then adaptation caused by a VC at the auditory level may simply not be transmitted through higher levels when testing is done on a CV series. At this time, a decision as to which of these two explanations is a better description of selective adaptation must await further research, possibly involving further spectral manipulations of the $\mathrm{CV}$ and VC syllables similar to those used by Bailey (1975) and Sawusch (1977).

One final point should be noted about the results from Experiments 1 and 2 . The only difference between the [ $\wedge \mathrm{d}] \mathrm{VC}$ syllable and the SEChirps was in the first formant and its final transition. This would seem to implicate the first formant as an indicator of the presence or absence of the place feature. In a model of speech perception, higher level processes which integrate information about the identity of a particular feature may also need to integrate information concerning whether the feature is present or not. This would act as a filtering mechanism and prevent the occurrence of spurious place responses in higher level mechanisms. In the context of the stimuli used in the present experiment, which contained a first formant with a relatively high steady-state frequency, an appropriate first-formant transition may be necessary for processing to take place in higher (possibly speech-specific) mechanisms.

In summary, the present experiments support the idea that selective adaptation to place at an auditory level of processing operates in a free-run mode without regard to the relative syllable positions of the critical information. Further, it was suggested that the failure of a VC syllable to adapt a CV series could be due to either of two possibilities, depending upon whether selective adaptation operates at one or two levels of processing during perception of the place feature in stop consonants. Within the two-level framework, cancellation due to adaptation effects in opposite directions at the two levels could account for these negative findings. In a one-level model, adaptation may simply not be transmitted through to higher level processing. Further research is needed to distinguish between these alternatives and to extend this line of reasoning to other features in speech.

\section{REFERENCE NOTES}

1. Bailey, P. J. Perceptual adaptation for acoustical features in speech. Speech perception: Report on research in progress in the Department of Psychology. Belfast: The Queen's University of Beltast, 1973, 2.2. 29-34.

2. Ganong, W. F. An experiment on "phonetic adaptation." RLE Progress Report, MIT, Cambridge, Massachusetts, 1975. 116. $206-210$.

3. Stevens, K. N. The potential role of property detectors in the perception of consonants. Paper presented at the Sym. posium on Auditory Analysis and Perception of Speech, August 1973. Leningrad, USSR.

4. Pisoni, D. B. Stages of processing in speech perception: F'ature unalysis. Paper presented at the Eighth International Congress of Phonetic Sciences, August 1975, Leeds, England.

5. Stevens, K. N., \& Blumstein, S. E. Context-independent cues for place of articulation in stop consonants. Paper presented at the 91 st meeting of the Acoustical Society of America, April 1976. Washington, D.C.

\section{REFERENCES}

Ades. A. E. How phonetic is selective adaptation? Experiments on syllable position and vowel environment. Perception \& Psychophysics, 1974, 16, 61-67.

ADEs. A. E. Adapting the property detectors for speech perception. In R. J. Wales \& E. Walker (Eds.), New approaches (1) Language mechanisms. Amsterdam: North-Holland. 1976.

BAILEY. P. J. Perceptual adaptation in speech: Some properties of detectors for acoustical cues to phonetic distinctions. Unpublished doctoral thesis. University of Cambridge. Cambridge, England. 1975.

Bi.limstein. S. E.. Stevens, K. N., \& Nigro, G. N. Property detectors for bursts and transitions in speech perception. Journal of the Acoustical Society of America, 1977. 61, $1.301-1313$

Cooper. F. S., Delattre, P. C., Liberman, A. M., Borst, J. M., $\&$ Gerstman. L. J. Sone experiments on the perception of synthetic speech sounds. Journal of the Acoustical Society of America, 1952, 24. 597-606.

COOPER. W. E. Adaptation of phonetic feature analyzers for place of artibulation. Journal of the Acoustical Society of America. 1974, 56. 617-627.

Cooper. W. E. Selective adaptation to speech. In F. Restle, R. M. Shittrin. N. J. Castellen, H. Lindman, \& D. B. Pisoni (Eds.). Cognitive theory (Vol. 1). Hillsdale. N.J: Erlbaun. 1975.

Cooper, W. E., \& Blumstein. S. E. A "labial" feature anilyzer in speech perception. Perception \& Psychophysics. 1974. 15. 591-600.

Dient. R. L. The effect of selective adaptation on the identitication of speech sounds. Perception \& Psychophysics, 1975. 17. $4 \times-52$.

Eimas. P. D., Cooper, W. E., \& Corbit, J. D. Some properties of linguistic feature detectors. Perception \& Psychophysics. 1973. 13, 247-252.

Eimas. P. D.. \& Corbit, J. D. Selective adaptation of linguistic feature detectors. Cognitive Psychology, 1973, 4, 99-109.

Hoffman. H. S. Study of some cues in the perception of the voiced stop consonants. Journal of the Acoustical Society of America. 1958. 30, 1035-1041.

HubeL, D. H.. \& WiEsel, T. N. Receptive tields and functional architecture in two nonstriate visual areas (18 and 19) of the cat. Joumal of Neurophysiology, 1965, 28, 229-289.

liberman. A. M.. Cooper. F. S., Shankweiler, D. P.. \& Studdert-Kennedy. M. Perception of the speech code. Psychological Review, 1967, 74, 431-461.

Lirerman, A. M.. Delattre. P. C.. Gerstman. L. J.. \& cooper. F. S. Tempo of frequency change as a cue for distinguishing classes of speech sounds. Jommal of Experimental Parchelengy. 1456. 52. 127-137. 
Pisoni. D. B.. \& TASH, J. B. Auditory property detectors and processing place features in stop consonants. Perception \& Psychophysics, 1975. 18, 401-408.

Sawusch, J. R. Peripheral and central processes in selective adaptation of place of articulation in stop consonants. Journal of the Acoustical Society of America, 1977, in press.

TARTter. V. C.. \& Eimas. P. D. The role of auditory and phonetic feature detectors in the perception of speech. Perception \& Psychophysics. 1975, 18. 293-298.

Weisstein, N. Beyond the yellow-Volkswagen detector and the grandmother cell: A general strategy for the exploration of operations in human pattern recognition. In R. L. Solso (Ed.). Contemporany issues in cognitive psychology. Washington. D.C: Winston, 1973

\section{NOTES}

1. This two-formant chirp was produced by a computer program designed to act as a parallel resonance synthesizer. This program was written by Dr. Dennis Klatt at MIT, who assisted in the preparation of this stimulus. His help is gratefully acknowledged.

2. Unless otherwise noted, all statistical tests were one-tailed $t$ tests for correlated measures.

3. The third curve (open triangles) on the right side of both Figures 3 and 4 represents cross-series adaptation with [^d] (Figure 3) and [bae] (Figure 4) in which the adaptor occurred 100 times in succession. This data is for the same two groups of five subjects who were asked back for a fourth day, during which their cross-series CV-VC adaptation and testing was repeated with more occurrences of the adaptor. This was done to match the procedure of Experiment 1 to that used by Pisoni and Tash (1975) and Pisoni (Note 4) as closely as possible. Despite this increased number of presentations of the adaptors, no adaptation effects were found.

4. $\mathrm{t}(8)=.519, \mathrm{p}<.5$, using a two-tailed $\mathrm{t}$ test for independent measures.

(Received for publication March 14, 1977; revision accepted July $1,1977$. ) 